\title{
Zur subjektiven Bedeutung von internationalem freiwilligen Engagement aus der Sicht ehemaliger Freiwilliger: Ein Literaturüberblick im Forschungsfeld
}

\author{
Franziska Müller \\ Doktorandin, Institut für Psychologie \\ Pädagogische Hochschule Karlsruhe | franziska.mueller@ph-karlsruhe.de
}

\section{Zusammenfassung}

Ein häufiges Ziel von geförderten internationalen Freiwilligendiensten ist die Fortführung von freiwilligem Engagement durch die Freiwilligen nach dem Dienst. In vielen Programmevaluierungen werden die Rückkehrer*innen daher auch nach ihrem aktuellen und zukünftigen Engagement befragt. Oft handelt es sich dabei um überwiegend quantitative Erhebungen. Der vorliegende Artikel zeigt auf der Grundlage eines Literaturüberblicks auf, dass eine Kombination aus einer entwicklungspsychologischen und einer biographieanalytischen Betrachtung zur Erfassung von Engagement dazu beitragen kann - zusätzlich zu den bereits erhobenen Zahlen -, aus der Innenperspektive der Freiwilligen retrospektiv nachzuvollziehen, wie sich das Engagement nach dem Dienst auch über einen längeren Zeitraum hinweg entwickelt hat. Hierfür werden Erkenntnisse aus Studien zur biographischen Einbettung von Engagement sowie aus der Entwicklungspsychologie auf den Kontext internationale Freiwilligendienste übertragen.

Schlagwörter: internationale Freiwilligendienste; subjektive Bedeutung; Biographie; Entwicklungspsychologie; Engagement nach dem Dienst; qualitative Forschung; biographisch-narrative Interviews

\begin{abstract}
Funded international voluntary services frequently aim at encouraging future volunteering after the service. Therefore, many evaluations focus on the returnees' further volunteering. These surveys are often predominantly quantitative-oriented evaluations. On the basis of a literature review the present article argues that a developmental psychological perspective together with a biographical research approach, in addition to the figures already collected, contributes to a deeper understanding of the longterm development of volunteering from the volunteers' internal perspective. Thus, findings from studies on the biographical embedding of volunteering and developmental psychology are transferred to the context of international voluntary services.
\end{abstract}

Keywords: International voluntary services; subjective meaning; biography; developmental psychology; volunteering after the service; qualitative research; biographical narrative interviews

\section{Einleitung}

Die unter anderem im Programmkonzept einiger geförderter internationaler Freiwilligendienste formulierten Erwartungen in Bezug auf Lern- und 
Entwicklungschancen für Freiwillige sind häufig sehr hoch. Dabei werden auch langfristige Wirkungen wie der Einfluss auf das spätere gesellschaftliche Engagement herausgestellt. Ein Ziel des Weltwärts-Programms beispielsweise besteht darin, dass sich die Freiwilligen auch nach ihrem Dienst in der entwicklungspolitischen Inlandsarbeit engagieren sollen (weltwärts 2019). Das Engagement der Rückkehrer*innen ist daher einer der Wirkungsbereiche, die in der Evaluierung des Programms betrachtet werden (Polak/Guffler/Scheinert 2017: vii). Wie dieses Engagement nach dem Dienst erhoben wird, ist sehr unterschiedlich. Auch die Ergebnisse der durchgeführten Studien variieren. Eine Evaluierung kommt zu dem Schluss, dass das Engagement der Freiwilligen nach dem Dienst vom Umfang her dem Engagement vor dem Dienst entspricht (Polak/Guffler/Scheinert 2017: 90). Eine weitere Studie verzeichnet einen leichten Rückgang des Engagements nach dem Dienst (Freudenberg u. a. 2017). Dieser Rückgang trotz hoher Engagementbereitschaft wird von Wissenschaftler*innen häufig mit der Umbruchsituation erklärt, in der sich viele Rückkehrer*innen nach ihrem Dienst befinden, zum Beispiel im Übergang zwischen Schule und Studium (Freudenberg u. a. 2017: 187; Fischer 2011: 62; Stern u. a. 2010: 73). Die Lebensphase der Freiwilligen nach dem Dienst scheint demnach einen entscheidenden Einfluss auf ihr Engagement zu haben. Es ist anzunehmen, dass eine grundsätzlich hohe Bereitschaft, aber geringe Möglichkeiten für Engagement bei den Freiwilligen zu Konflikten führt. Die vorliegenden quantitativen Studien sind diesbezüglich wenig aussagekräftig. Qualitative Studien, die detailliert nicht nur die Phase nach dem Dienst, sondern auch die Entwicklung des Engagements über mehrere Jahre hinweg in den Blick nehmen, lassen sich bisher kaum finden. Zu nennen ist hier beispielsweise Kühn, die in ihrer Studie die Daten von zehn Weltwärts-Freiwilligen analysiert hat, die sie mittels leitfadengestützter, narrativer Interviews zu zwei Zeitpunkten befragt hat, vor der Ausreise und circa drei Monate nach dem Dienst (Kühn 2015: 85 ff.). Auch Mundorf hat qualitativ mit problemzentrierten, teilstrukturierten Interviews gearbeitet (Mundorf 2000: 48 f.). In ihrer Studie stehen die Ergebnisse der retrospektiven Befragung von acht Teilnehmenden eines christlichen Freiwilligendienstes im Ausland im Mittelpunkt, deren Freiwilligendienst zwischen zweieinhalb und fünf Jahren zurücklag (Mundorf 2000: 52 ff.). Bei beiden Studien ist das Engagement nach dem Dienst allerdings nur ein Aspekt unter vielen.

Im vorliegenden Beitrag werden die genannten Studien zu Freiwilligendiensten, die sich unter anderem auf das Engagement nach dem Dienst beziehen (Kapitel 2), mit entwicklungspsychologischen Studien (Kapitel 3) sowie Studien zu Engagement im Rahmen der Biographie (Kapitel 4) zusammengeführt. Im Mittelpunkt steht dabei die folgende Frage: Was können eine entwicklungspsychologische sowie eine biographieanalytische Perspektive zum Stand der Forschung zu Engagement nach dem Dienst beitragen? Es geht dabei nicht nur um das Rückkehrengagement, 
sondern generell um das Engagement der Freiwilligen. In Kapitel 5 werden - aus den Erkenntnissen der oben genannten Studien abgeleitet - die Stärken der Kombination aus entwicklungspsychologischer und biographieanalytischer Perspektive für die Erforschung der subjektiven Bedeutung von internationalem freiwilligen Engagement aus der Sicht ehemaliger Freiwilliger zusammengefasst. Die hier vorliegenden Überlegungen sind Teil meines laufenden Dissertationsprojektes, ${ }^{1}$ das ebenfalls in Kapitel 5 kurz vorgestellt wird.

Im Rahmen meiner Recherche zu internationalem freiwilligem Engagement bin ich sehr schnell auf die Schriftenreihe Interdisziplinäre Studien zu Freiwilligendiensten und die darin publizierten Autor*innen gestoßen, wie beispielsweise Haas (2012), sowie - über die Schriftenreihe hinaus - auf weitere wissenschaftliche Publikationen zu Freiwilligendiensten zum Beispiel von Fischer (2011), Mangold (2014) sowie Fischer und Haas (2018). Die hier vorgestellten Studien gehen maßgeblich auf die Lektüre dieser Arbeiten sowie deren Quellen zurück.

\section{Das Engagement nach dem Dienst in Zahlen}

Die vier ausgewählten Studien zu Weltwärts erfassen das Engagement nach dem Dienst überwiegend mittels quantitativer Methoden. ${ }^{2}$ Drei der Studien wurden im Rahmen des Qualitätssystems von Weltwärts von unabhängigen Gutachter*innen bzw. Evaluierungsinstituten durchgeführt (uzbonn 2018; Polak/Guffler/Scheinert 2017; Stern u. a. 2011). Die vierte Studie (Freudenberg u. a. 2017) entstand im Rahmen eines Forschungsprojektes an der Zeppelin Universität.

In der jährlichen Freiwilligenbefragung zum Weltwärts-Programm werden mittels Fragebögen jeweils die Freiwilligen befragt, die in dem entsprechenden Jahr ihren Dienst beendet haben. Bei der Befragung von 2017 wurden die Daten von 2.147 Freiwilligen ausgewertet (uzbonn 2018: 2). Die meisten der Befragten hatten den Freiwilligendienst direkt nach der Schule angetreten und waren zum Zeitpunkt der Befragung durchschnittlich 19 Jahre alt (uzbonn 2018: 2 f.). In der Freiwilligenbefragung wurde unter anderem nach dem aktuellen Engagement sowie der Engagementbereitschaft der Rückkehrer*innen gefragt. Engagement wird definiert als ,jegliche Tätigkeit, die nicht auf Bezahlung basiert, sondern auf die Förderung des Gemeinwohls ausgerichtet ist - und nicht nur auf den entwicklungspolitischen Bereich beschränkt [ist]“ (uzbonn 2018: 17). Folgende Ergebnisse wurden festgehalten: Von den Rückkehrer*innen haben sich 39 Prozent zum Zeitpunkt der Befragung freiwillig engagiert. 46 Prozent der Befragten gaben an,

1 Arbeitstitel: „Die Bedeutung von internationalem freiwilligen Engagement für ehemalige Freiwillige aus entwicklungspsychologischer Perspektive“.

2 Auch wenn in zwei Evaluierungen (Polak/Guffler/Scheinert 2017; Stern u. a. 2011) zusätzlich Interviews bzw. Gruppendiskussionen angewandt wurden, stützen sich die meisten hier aufgeführten Ergebnisse auf die Fragebogen-Erhebungen. 
sich zukünftig sehr sicher bzw. wahrscheinlich zu engagieren (uzbonn 2018: 17). Die Verfasser*innen der Evaluierung interpretieren dies insgesamt als positiv. Allerdings kann hier angemerkt werden, dass von der Engagementbereitschaft nicht ohne Weiteres auf das tatsächliche Engagement geschlossen werden kann, wie Fischer (2011: 62) bereits herausgestellt hat.

Differenziertere Ergebnisse liefert die vom Deutschen Evaluierungsinstitut der Entwicklungszusammenarbeit (DEval) durchgeführte Evaluierung, bei der unter anderem die Wirkungen des Weltwärts-Programms auf die Freiwilligen im Mittelpunkt standen (Polak/Guffler/Scheinert 2017: vii). Zur Erfassung des Engagements der Rückkehrer*innen in Deutschland wurde das Engagement vor dem Dienst mit dem Engagement danach verglichen. Dafür wurden die Daten von insgesamt 2.829 ausreisenden Freiwilligen sowie Rückkehrer*innen ausgewertet (Polak/Guffler/Scheinert 2017: 25; 89 f.). Durch einen Vergleich mit der bevölkerungsrepräsentativen Zielgruppe wird deutlich, dass Weltwärts-Freiwillige schon vor dem Freiwilligendienst deutlich engagierter sind als Personen, die keinen Freiwilligendienst leisten. Zwischen dem Engagement der Freiwilligen vor dem Dienst und dem Engagement danach konnte kein zahlenmäßiger Unterschied festgestellt werden. Es ändert sich aber die Art des Engagements der Freiwilligen, das nach dem Dienst tendenziell mehr Bezug zu entwicklungspolitischen Themen aufweist (Polak/Guffler/Scheinert 2017: 90 f.).

Freudenberg u. a. (2017) haben in ihrer Studie mittels Online-Fragebögen ehemalige Weltwärts-Freiwillige befragt, die im Durchschnitt 23 Jahre alt waren (Freudenberg u. a. 2017: 179 ff.). Es wurden Daten von 389 Personen ausgewertet. Die meisten studierten zum Zeitpunkt der Befragung. Freudenberg u. a. ermitteln einen Rückgang des Engagements von 82 Prozent vor dem Dienst auf 72,2 Prozent nach dem Dienst. Als Gründe, sich nicht zu engagieren, nannten die Freiwilligen unter anderem Zeitmangel (Freudenberg u. a. 2017: 180 ff.). Die Autor*innen der Studie weisen in ihrer zusammenfassenden Erörterung auf die Zeit unmittelbar nach dem Dienst hin, in der viele der Freiwilligen mit einem Wechsel zum Studienort beschäftigt sind und dadurch Engagement möglicherweise erschwert wird (Freudenberg u. a. 2017: 187).

Ein Ziel der Evaluierung von Stern u. a. war ebenfalls die Überprüfung von Wirkungen des Weltwärts-Programms (Stern u. a. 2011: 1). Es lagen insgesamt 4.890 ausgefüllte Online-Fragebögen von aktuellen und ehemaligen Freiwilligen vor (Stern u. a. 2011: 2). Für den vorliegenden Artikel ist besonders eines ihrer Ergebnisse grundlegend: Sie konnten feststellen, dass sich das Engagement der Weltwärts-Rückkehrer*innen im Laufe der Zeit leicht erhöht hat. In einem Zeitraum von 14 Monaten nach der Rückkehr waren 61 Prozent der Rückkehrer*innen engagiert. Nach 14 Monaten stieg das Engagement auf 69 Prozent an (Stern u. a. 2011: 73). Ähnlich wie Freudenberg u. a. kommen auch Stern u. a. (2011: 73) zu der 
Erklärung, dass die neue Lebensphase und die damit verbundenen Änderungen wie ein Umzug für das Engagement der Rückkehrer*innen hinderlich sein können, auch wenn diese sich engagieren möchten.

Aus den oben genannten Studien kann abgeleitet werden, dass das Engagement der Weltwärts-Freiwilligen nach dem Dienst im Vergleich zu ihrem Engagement vor dem Dienst zahlenmäßig nicht zunimmt bzw. (vorläufig) sogar etwas zurückgehen kann. Es gibt außerdem Anzeichen dafür, dass sich die Anforderungen der Lebensphase, in der sich die Freiwilligen nach dem Dienst häufig befinden, zumindest kurzfristig negativ auf eine Fortführung ihres Engagements auswirken. Dies wird durch die Ergebnisse der oben bereits erwähnten Studie von Kühn (2015) bestätigt. In den Erzählungen der Befragten nach dem Dienst hat Engagement aufgrund anderer anstehender Aufgaben wie der Berufswahl oder der Wohnungssuche nur eine geringe Priorität (Kühn 2015: 177). Bei Mundorf gibt zwar ein Großteil der Befragten in den Kurzfragebögen an, in Kirchengemeinden ehrenamtlich aktiv zu sein, in den Interviews selbst gehen die befragten ehemaligen Freiwilligen aber nur in einigen wenigen Fällen auf ihr Engagement nach dem Dienst ein, sodass darüber wenig ausgesagt werden kann (Mundorf 2000: 115 f.).

Eine entwicklungspsychologische Sicht, die im Folgenden ausgeführt wird, kann weitere Erklärungsmöglichkeiten für den in Zahlen geschilderten Verlauf des Engagements liefern.

\section{Eine entwicklungspsychologische Betrachtung von freiwilligem Engagement im aufkommenden und jungen Erwachsenenalter}

Wie oben gesehen, sind die Weltwärts-Freiwilligen bei ihrer Rückkehr durchschnittlich um die 20 Jahre alt. Aus entwicklungspsychologischer Perspektive markiert dieses Alter die Grenze zum jungen Erwachsenenalter, das sich circa bis zum 30. Lebensjahr erstreckt. ${ }^{3}$ Da ein Großteil der Freiwilligen nach dem Freiwilligendienst studiert, liegt es nahe, dass hier die Phase der Emerging Adulthood (,,aufkommendes Erwachsenenalter") zutrifft, die von Arnett (2000) geprägt wurde (Freund/Nikitin 2012: 263 f.). Diese Phase zwischen Adoleszenz und Erwachsenenalter bezieht sich auf das Alter zwischen 18 und 25 Jahren. Sie zeichnet sich durch ein hohes Maß an Exploration verschiedener Möglichkeiten und einer weitgehenden Unabhängigkeit von sozialen Erwartungen aus (Arnett 2000: 469). Dem Autor zufolge findet in dieser Phase, neben der Adoleszenz, ein Großteil der Identitätsentwicklung statt (Arnett 2000: 473). Auf die positiven Auswirkungen von gesellschaftlichem Engagement auf die Identitätsentwicklung von Jugendlichen wurde bereits in mehreren Studien hingewiesen

3 Über die Schwierigkeiten einer genauen Zuordnung von Lebensphasen zu einem bestimmten Alter siehe Freund und Nikitin (2012: 260). 
(Überblick in Buhl/Kuhn 2005). Es ist anzunehmen, dass auch in der Phase der Emerging Adulthood freiwilliges Engagement als Möglichkeit des sich Ausprobierens weiterhin Bestand im Leben der Freiwilligen haben wird, wenn auch die Ausrichtung möglicherweise eine etwas andere sein wird. Während für Jugendliche unter anderem die Ablösung vom Elternhaus bzw. Selbstfindung und berufliche Orientierung wichtige Motive für die Teilnahme an einem internationalen Freiwilligendienst sind (Krettenauer/Gudulas 2003: 225 f.), dienen Praxiserfahrungen ${ }^{4}$ Älteren zum Beispiel zielgerichteter als Vorbereitung auf den zukünftigen Beruf (Arnett 2000: $473 \mathrm{f}$.), aber auch ganz generell dem Sammeln unterschiedlicher Erfahrungen (Arnett 2000: 474).

Eine Konsequenz dieser Phase ist, dass die für das junge Erwachsenenalter typischen Entwicklungsaufgaben ${ }^{5}$ wie Aufbau einer Partnerschaft, Familiengründung sowie der Einstieg in das Berufsleben in eine spätere Phase verschoben werden. Dies führt dazu, dass sich die eigentliche Phase des jungen Erwachsenenalters verkürzt und umso fordernder wird. Daher wird sie auch als „Rushhour“ des Lebens bezeichnet (Freund/Nikitin 2012: 264;281). Für freiwilliges Engagement ist in dieser Phase dementsprechend wenig Zeit. Olk hat bereits auf diesen Zusammenhang zwischen den Anforderungen in dieser Phase und dem Rückgang von Engagement hingewiesen (2010: 648).

Zusammenfassend kann festgehalten werden, dass es aus entwicklungspsychologischer Sicht eine ideale Passung zwischen den Bedürfnissen von Jugendlichen hinsichtlich Identitätsentwicklung und den Angeboten eines internationalen Freiwilligendienstes zu geben scheint. Weiterhin ist anzunehmen, dass auch in der darauffolgenden Phase, der Emerging Adulthood, freiwilliges Engagement als Möglichkeit des sich Ausprobierens im Leben der Freiwilligen Bestand haben wird. Ein Rückgang des Engagements wäre dann nur temporär. Dies entspricht auch den Zahlen des Freiwilligensurveys, wonach die Gruppe der 20- bis 24-Jährigen ähnlich stark engagiert ist wie die Gruppe der 14- bis 19-Jährigen, die sich zahlenmäßig gemessen an der in Deutschland lebenden Bevölkerung insgesamt am meisten engagieren. Die beiden darauffolgenden Gruppen der 25- bis 29-Jährigen sowie der 30- bis 34-Jährigen allerdings engagieren sich im Vergleich merklich weniger ${ }^{6}$ (Vogel u. a. 2017: 103 f.).

Einen weiteren Einblick in mögliche Gründe für die Fortführung oder die Aufgabe von Engagement geben biographieanalytische Studien. Dies wird im Folgenden ausgeführt.

4 Arnett bezieht sich hier nicht explizit auf freiwilliges Engagement.

5 Mit Entwicklungsaufgaben sind ,in einem bestimmten sozial-kulturellen Kontext geteilte soziale Erwartungen, was man zu einem bestimmten Alter tun und erreichen sollte“ (Freund/Nikitin 2012: 262 f.) gemeint. Freund und Nikitin gehen auf die soziologische Kritik an Entwicklungsaufgaben ein, betonen aber auch deren breite Gültigkeit (2012: 263).

6 Es wird dort auf weitere Faktoren wie bspw. Geschlecht und Bildung eingegangen, die auch im Dissertationsprojekt der Autorin dieses Artikels näher betrachtet werden. In diesem Beitrag steht allerdings das Alter im Vordergrund. 


\section{Freiwilliges Engagement über die gesamte Biographie hinweg}

Der Verlauf von Engagement im Erwachsenenalter bzw. über die gesamte Biographie hinweg wurde in mehreren Studien betrachtet, von denen zwei im Folgenden vorgestellt werden. Auch wenn es in diesen Arbeiten nicht spezifisch um Freiwilligendienste geht, können daraus dennoch wichtige Erkenntnisse für die Entwicklung von Engagement nach dem Dienst abgeleitet werden.

Jakob hat in ihrer Studie ehrenamtliches Engagement als Teil der Biographie analysiert und dazu mit elf ehrenamtlich tätigen Personen autobiographisch-narrative Interviews geführt. Die Befragten waren zwischen 25 und 75 Jahre alt, wobei die meisten über 40 waren (Jakob 1993: 30 ff.). Das Ergebnis ihrer Studie sind fünf verschiedene Typen ehrenamtlichen Engagements. Bei allen bis auf einen Typ ist das Engagement für die Befragten sinn- und identitätsstiftend (Jakob 1993: 226). Für den vorliegenden Artikel sind zwei Typen von besonderem Interesse: „Soziale Ehrenamtlichkeit als Instrument der Suche nach biographischer Orientierung“ und „Die Realisierung eigener biographischer Themen mit ehrenamtlichen Tätigkeiten“ (Jakob 1993: 118 ff.). Beide Typen zeichnen sich durch ausgeprägte Wandlungsprozesse in der Biographie aus (Jakob 1993: 232 f.). Unter Bezug auf soziologische Theorien stellt Jakob dar, wie sich Umbrüche kultureller und sozialer Art auf das Leben und somit auch das ehrenamtliche Engagement der Befragten auswirken (Jakob 1993: 272 ff.). Die Autorin kommt zu dem Schluss, dass das Ehrenamt durch Biographisierungsprozesse nicht mehr selbstverständlich ein Leben lang ausgeführt wird, sondern je nach Lebensphase „für die Realisierung selbstbezogener Aspekte in Anspruch genommen [wird]“ (Jakob 1993: 281 f.). Ihr zufolge ist eine der zentralen Voraussetzungen für die Aufnahme bzw. Fortführung von Engagement, wie es auf die beiden oben genannten Typen zutrifft, die „,biographische Passung“ (Jakob 1993: 281).

In drei der unter Kapitel 2 genannten Studien (Freudenberg u. a. 2017; Kühn 2015; Stern u. a. 2011) wird darauf hingewiesen, dass die Zeit nach dem Dienst für die Freiwilligen häufig mit einem Wechsel des Wohnorts verbunden ist. Es kann also davon ausgegangen werden, dass auch die Biographien der Freiwilligen eher durch Flexibilität und Wandel als durch Kontinuität geprägt sind.

Im Mittelpunkt der qualitativen Arbeit von Benedetti (2015) steht die Frage nach der Relation von Erwerbsarbeit und gesellschaftlichem Engagement. Dies ist für den vorliegenden Beitrag insofern von Interesse, als dass, wie oben dargestellt, die berufliche Festigung eine wichtige Aufgabe im jungen Erwachsenenalter ist. Benedettis Arbeit zugrunde liegen die Daten von sieben Personen, von denen sechs Personen im Abstand von 20 bis 25 Jahren mittels offen-narrativer, thematisch 
fokussierter Doppelinterviews befragt wurden (Benedetti 2015: 92 ff.). ${ }^{7}$ Eine wichtige Erkenntnis der Arbeit betrifft die Dynamizität gesellschaftlichen Engagements, die sich aus der starken Abhängigkeit von individuellen und gesellschaftlichen Kontexten ergibt. Diese umfassen unter anderem die bildungsbiographische Orientierung, zeitliche und physische Ressourcen, aktuelle Diskurse sowie soziale und politische Gruppierungen (Benedetti 2015: 376 f.). Die Befragten hatten zum Zeitpunkt des zweiten Interviews weder die gleiche Arbeit noch das gleiche Engagement wie beim ersten Interview inne. Die hohe Veränderbarkeit der Relation von Erwerbsarbeit und Engagement kann auch zu einer Veränderung der subjektiven Bedeutung des gesellschaftlichen Engagements im Laufe der Zeit führen. Als existenziell wahrgenommene Engagementmöglichkeiten wurden in der Retrospektive nicht mehr als solche wahrgenommen (Benedetti 2015: 386 ff.).

Beiden Studien ist zu entnehmen, dass sich freiwilliges Engagement im Verlauf der Biographie einer engagierten Person stetig wandelt und das Fortführen von Engagement von vielen unterschiedlichen individuellen und gesellschaftlichen Aspekten abhängt. Um mehr über die subjektive Bedeutung von Engagement und die Entwicklung über einen längeren Zeitraum hinweg herauszufinden, ist eine biographische Betrachtung daher sehr aufschlussreich.

\section{Fazit und Ausblick}

Während also eine entwicklungspsychologische Perspektive den Blick für bestimmte Lebensphasen und deren sozial erwartete Anforderungen sowie persönliche Entwicklungsprozesse schärft, bietet ein biographischer Ansatz Möglichkeiten, um auch individuelle Themen zu erfassen, die gegebenenfalls aus weiter zurückliegenden Lebensabschnitten herrühren, aber mit dem Engagement in Verbindung stehen. Das Anliegen dieses Beitrags ist es aufzuzeigen, dass eine Kombination aus entwicklungspsychologischer und biographieanalytischer Betrachtung des Engagements der Freiwilligen dazu beiträgt, mehr über die subjektive Bedeutung von Engagement für die Freiwilligen herauszufinden und damit auch über mögliche Gründe, warum Engagement beibehalten oder (vorübergehend) beendet wird. Dies kann weitere Erkenntnisse liefern, wie Engagement nach dem Dienst noch gezielter unterstützt werden kann. Gerade für die Zeit unmittelbar nach dem Dienst, aber auch im Hinblick auf eine Entwicklung des Engagements über Jahre hinweg kann dies Anhaltspunkte bieten in Bezug auf zum Engagement konkurrierende Prioritäten. Die Umbruchsituation zum Beispiel durch einen Umzug kann ein Aspekt sein, warum Engagement aufgegeben wird. Sollte es für die Person aber beispielsweise eine biographisch wichtige Rolle

7 Die Interviews stammen aus dem Gesamtsample des DFG-Forschungsprojektes "Prekäre Kontinuitäten" (Benedetti 2015: 29 ff.). Ein Kriterium der Auswahl der Interviews war die Thematisierung von gesellschaftlichem Engagement im Erwachsenenalter (Benedetti 2015: 93 f.). 
spielen, so wird sie sich auch nach einem Umzug gegebenenfalls in veränderter Form weiter engagieren. Die Kombination beider Perspektiven verspricht folglich ein umfassendes Bild von Engagement aus der Innenperspektive der Freiwilligen und stellt somit eine Bereicherung zum aktuellen Stand der Forschung dar.

Eine empirische Untersuchung der hier dargestellten theoretischen Erkenntnisse erfolgt im Rahmen meines derzeit laufenden Dissertationsprojektes. Zur Erfassung subjektiver Bedeutungen von Engagement sowie von Entscheidungen für oder gegen die Fortführung von Engagement und möglicher Konflikte diesbezüglich habe ich mittels biographisch-narrativer Interviews in Anlehnung an Schütze (2016) bisher elf ehemalige Freiwillige nach ihrer Lebensgeschichte, mit Fokus auf ihr Engagement, befragt. ${ }^{8}$ Neben Freiwilligendiensten im Ausland beschäftige ich mich auch mit internationalem Engagement im Hochschulkontext und in internationalen Hilfsorganisationen. Die Dauer des Auslandsaufenthaltes der befragten Freiwilligen variiert zwischen drei Wochen und eineinhalb Jahren. Gemeinsam ist allen Befragten, dass sie zum Zeitpunkt des Interviews bereits berufstätig waren. Das Durchschnittsalter liegt zwischen Ende 20 und Anfang 30. Eine systematische Analyse der geführten Interviews wird aktuell durchgeführt.

\section{Literaturverzeichnis}

Arnett, Jeffrey J. (2000): Emerging adulthood. A theory of development from the late teens through the twenties, in: American Psychologist, 55. Jg., Heft 5, S. 469480.

Benedetti, Sascha (2015): Engagement, Biographie und Erwerbsarbeit. Eine biographieanalytische Studie zur subjektiven Bedeutung gesellschaftlichen Engagements, Wiesbaden.

Buhl, Monika und Hans-Peter Kuhn (2005): Erweiterte Handlungsräume im Jugendalter: Identitätsentwicklung im Bereich gesellschaftlichen Engagements, in: Entwicklung in sozialen Beziehungen. Heranwachsende in ihrer Auseinandersetzung mit Familie, Freunden und Gesellschaft, hrsg. von Beate H. Schuster, Hans-Peter Kuhn und Harald Uhlendorff, Stuttgart, S. 217-237.

Fischer, Jörn (2011): Freiwilligendienste und ihre Wirkung - Vom Nutzen des Engagements, in: Aus Politik und Zeitgeschichte, 61. Jg., Heft 48, http://www.bpb.de/apuz/59669/freiwilligendienste-und-ihre-wirkung-vom-nutzen-des-engagements? $p=$ all (22.02.2019).

Fischer, Jörn und Benjamin Haas (2018): „Prägende Erfahrungen“ - und dann? Internationale Freiwilligendienste und das Engagement danach, in: Sozial Extra, 42. Jg., Heft 2, S. 51-54.

Freudenberg, Nora, Sophia Gläser, Julian Kohlruss, Pius Ohr und Louisa-Madeline Singer (2017): Das Engagement danach - Einflussfaktoren aus dem Freiwilligendienst am Beispiel von weltwärts, in: Voluntaris - Zeitschrift für Freiwilligendienste, 5. Jg., Heft 2, S. 172-190.

Freund, Alexandra M. und Jana Nikitin (2012): Junges und mittleres Erwachsenenalter, in: Entwicklungspsychologie, 7. Aufl., hrsg. von Wolfgang Schneider, Ulman Lindenberger, Weinheim-Basel, S. 259-282.

8 Details zum methodischen Vorgehen finden sich in Müller (2019). 
Haas, Benjamin (2012): Ambivalenz der Gegenseitigkeit. Reziprozitätsformen des weltwärtsFreiwilligendienstes im Spiegel der Postkolonialen Theorie, Köln.

Jakob, Gisela (1993): Zwischen Dienst und Selbstbezug. Eine biographieanalytische Untersuchung ehrenamtlichen Engagements, Opladen.

Krettenauer, Tobias und Niki Gudulas (2003): Motive für einen Freiwilligendienst und die Identitätsentwicklung im späten Jugendalter: Eine empirische Untersuchung zur Lebenslaufcharakteristik „,neuen sozialen Engagements“, in: Zeitschrift für Entwicklungspsychologie und Pädagogische Psychologie, 35. Jg., Heft 4, S. 221-228.

Kühn, Hannah M. (2015): Da entwickelt sich was! Individuelle Lernprozesse im entwicklungspolitischen Freiwilligendienst "weltwärts" in Benin, Köln.

Mangold, Katharina (2014): Inbetweenness als Möglichkeit transnationaler Wissensproduktion? Junge Erwachsene im Internationalen Freiwilligendienst - zwischen Herstellung von Stereotypen und transnationalem Wissen, in: Orte transnationaler Wissensproduktion. Sozial- und kulturwissenschaftliche Schnittmengen, hrsg. von Désirée Bender, Annemarie Duscha, Tina Hollstein, Lena Huber, Kathrin Klein-Zimmer und Caroline Schmitt, Weinheim-Basel, S. 128-152.

Mundorf, Margret (2000): Christliche Freiwilligendienste im Ausland: Lernprozesse und Auswirkungen auf die Lebensentwürfe junger Menschen. Eine qualitative Studie, Münster.

Müller, Franziska (2019): Using a Qualitative Research Approach to Investigate Identity Development in the Context of International Volunteering Experience, in: Diversity in Qualitative Research, 2., erw. Aufl., hrsg. von Günter L. Huber, Tübingen, S. 31-46, http://www.qualitative-psychologie.de/NEXUS/nexus-vol15.pdf (14.02.20).

Olk, Thomas (2010): Bürgerschaftliches Engagement im Lebenslauf, in: Soziale Lebenslaufpolitik, hrsg. von Naegele, Gerhard, Wiesbaden, S. 637-672.

Polak, Tobias, Kerstin Guffler und Laura Scheinert (2017): weltwärts-Freiwillige und ihr Engagement in Deutschland, Deutsches Evaluierungsinstitut der Entwicklungszusammenarbeit (DEval), Bonn, https://www.deval.org/files/content/Dateien/Evaluierung/ Berichte/2018/DEval_weltwaerts_Bericht_DE_web_links_final.pdf (22.02.2019).

Schütze, Fritz (2016): Biographieforschung und narratives Interview, in: Sozialwissenschaftliche Prozessanalyse. Grundlagen der qualitativen Sozialforschung, hrsg. von Werner Fiedler und Heinz-Hermann Krüger, Opladen-Berlin-Toronto, S. 55-73.

Stern, Tobias, Jan O. Scheller, Juliane Feix, Judith Käser-Erdtracht, Matias Krämer d'Oliveira, Katharina Plutter, Lennart Raetzell und Grit Würmseer (2011): Der entwicklungspolitische Freiwilligendienst „weltwärts“, Band I: Hauptbericht. Unveröffentlichter Evaluierungsbericht, Bonn.

Uzbonn (2018): Ergebnisse der Freiwilligenbefragung 2017 zum weltwärts-Programm, www. weltwaerts.de/de/publikation-detail.html?id=424 (22.02.2019).

Vogel, Claudia, Christine Hagen, Julia Simonson und Clemens Tesch-Römer (2017): Freiwilliges Engagement und öffentliche gemeinschaftliche Aktivität, in: Freiwilliges Engagement in Deutschland. Der Deutsche Freiwilligensurvey 2014, hrsg. von Julia Simonson, Claudia Vogel und Clemens Tesch-Römer, Berlin, S. 85-147.

Weltwärts (2019): Programm: Der entwicklungspolitische Freiwilligendienst stellt sich vor, www.weltwaerts.de/de/programm.html (22.02.2019). 